\title{
Biofimls Formation in Nanofiltration Membranes
}

\author{
Francisca Pessôa de França ${ }^{1 *}$, Renata Oliveira da Rocha Calixto ${ }^{1}$, Virgínia Carmen Rocha Bezerra ${ }^{1}$, Priscila \\ Martins da Rocha ${ }^{1}$, Edna Teresa Ruas Bastos ${ }^{2}$ and Celina Cândida Ribeiro Barbosa ${ }^{2}$ \\ 1. Chemistry School, Federal University of Rio de Janeiro, Rio de Janeiro 21941-909, Brazil
}

2. Nuclear Engineering Institute, Federal University of Rio de Janeiro, Rio de Janeiro 21941-906, Brazil

\begin{abstract}
Technological advances in the past 30 years have boosted the use of PSM (membrane separation processes), important for its efficiency and flexibility of operation. These processes can be used in many types of separation, with some advantages over the usual separation processes. NF (nanofiltration) is a membrane separation technique, which has properties intermediate between reverse osmosis and ultrafiltration in terms of separated species, because the average of the pores is in the range of $1 / 2$ to $10 \mathrm{~nm}$, and the separation occurs in function of load and size of the species. Usually removes species in solution with an effective diameter of about $1 \mathrm{~nm}$ or larger and multivalent ions to a greater extent than monovalent ions. The objective was to study the formation of biofouling on the surface of commercial nanofiltration membrane (Osmonics/GE) and surface membrane synthesized in our laboratory. The study was conducted in permeation system with filtration cell with tangential displacement of 15 bar for 8 days flow. DBNPA (2,2-dibromo-3-nitrilopropionamide) was used as a biocide agent, and an anti-fouling, in concentrations of 5 and 300 ppm, respectively, added to the water coming from the Beach Sea Galleon, RJ. The results demonstrated that there was no change in the flow and rejection of sulphate ions, even in the presence of anti-fouling. The count of aerobic, anaerobic and BRS (sulfate reducing bacteria) in seawater before and after using the DBNPA showed efficiency in controlling these groups of microorganisms and biofouling microbial consortium consisting of the existing in seawater.
\end{abstract}

Key words: Nanofiltration membrane, biofouling, biocide, sulfate reducing bacteria, seawater.

\section{Introduction}

Technological advances in the past 30 years have boosted the use of PSM (membrane separation processes), which have became important, with efficiency and flexibility of operation. The membrane processes can be used in various types of separation, constituting an innovation, with some advantages over the usual separation methods, such as low energy demand better quality of final product and increased operational flexibility, the membrane processes promote separation without any phase shift component, which means a considerable saving of energy [1].

$\mathrm{NF}$ (nanofiltration) is a membrane process with separation characteristics between RO (reverse osmosis) and UF (ultrafiltration) membranes. The average pore diameter of these membranes is in the

\footnotetext{
*Corresponding author: Francisca Pessôa de França, D.Sc/Ph.D., research fileds: microbiology and biochemical engeneering. E-mail: fpfranca@eq.ufrj.br.
}

range of $1 / 2$ to $10 \mathrm{~nm}$, and membranes have charges when in aqueous solution. The separation is a function of the load and the size of the species. Generally the nanofiltration removes species in solution that has an effective diameter of about $1 \mathrm{~nm}$ or larger, and remove multivalent ions to a greater extent than monovalent ions [2]. This technology has been widely used in water treatment, however, a major challenge of these processes is the accumulation of material on the membrane surface or within the pores, this phenomenon is commonly known as fouling. Fouling is a limiting phenomenon for the operations of membrane separation and is a function of several parameters: the membrane characteristics, dietary patterns and hydrodynamic conditions of the system. This phenomenon can significantly reduce the permeate flux, increase operating costs and shorten the life of the membrane [3, 4].

The main cause of fouling is the adsorption of inorganic and organic materials on the membrane 
surface $[5,6]$. It depends on the hydrophobicity of the membrane and the feed components, and when loaded are filtered compounds, such as salts or organic acids, the surface charge of the membrane is affected largely acting in their interaction. Both the electrostatic repulsion as attraction is used to explain the decline of flux and fouling of components loaded [7-9].

The biofouling and its effects are easily occurred through the initial process such as the location of the surface body holding surface and adhesion. The dimensions of fouling organisms causing sedimentation during its stages (bacteria, cells, spores) are typically $500 \mathrm{~nm}$ to $100 \mathrm{~nm}$, but the surface via recognition of the relevant sensory structures probably occurs at much smaller length scales (nm or even smaller) level. This process is a hierarchical event in time, because the "clean" surface can be rapidly enriched by chemicals in marine environments, allowing microbial adhesion and proliferation with emphasis on bacteria and microalgae [10]. The bacteria are able to colonize the surfaces can present extreme conditions, such as temperatures from $-12{ }^{\circ} \mathrm{C}$ to $110^{\circ} \mathrm{C}$ and $\mathrm{pH}$ between 0.5 and 13. Bacteria embedded in the biofilms are more resistant to biocides than bacteria dispersed state. This is related the presence of biopolymers which prevents the penetration of biocides in biofilms [11]. The colonization of different species is often synergistic in the biofilm and in the case of etched surface, bacterial colonization, adhesion of unicellular algae and the release of spores of certain encrusting algae often require only a few minutes to a few hours. In these early stages of biofilm formation, physical interactions are the forces experienced by the first fouling organisms and adherence is often determined by the physicochemical surface properties [10].

The biofouling occurs despite the use of pre-treatment and the addition of disinfectants such as chlorine systems, but are often not effective, requiring replacement of the material [11].
This work aimed to study the formation of biofilm on the surface of nanofiltration membranes (DK, Osmonics/GE) and membranes fabricated in the laboratory containing DDS (bis-4-aminophenyl-sulfone) embedded in its selective layer using seawater in nature and the formation of biofilm on these same media in the presence of DBNPA (2,2-dibromo-3-nitrilopropionamide) and an antifouling.

\section{Materials and Methods}

\subsection{Sample}

The seawater was subjected to successive filtration using filter paper with a pore size of $80 \mu \mathrm{m}$ and a pore size of $5 \mu \mathrm{m}$, before beginning the experiment.

\subsection{Membranes and System Operation}

The membranes used in this study were provided by DK/Osmonics/GE) and synthesized in our laboratory (containing in its composition DDS (bis-4-aminophenyl-sulfone).

Experiments to evaluate of the membranes were performed in a permeation cell with a displacement tangential flow mark Flutrol (Fig. 1). This system consists of a permeation cell, high pressure pump, pressure gauge, flow meter, valve and reservoir to supply water to a maximum volume of $10 \mathrm{~L}$. The permeable membrane area is $119 \mathrm{~cm}^{2}$ and is represented by any surface in contact with the feed stream. The pressure used was 15 bar. The experiment was performed on 8 days of operation, and water changes were made every two days of operation, totaling 4 water changes.

During the procedure, samples of water permeated through the membrane (permeate) to calculate the percentage rejection of sulfate ions was collected. These analyzes were performed at the Ion Chromatograph Dionex ICS 1000, Ion Pac AS23 anion column, specifically for analysis of anions in seawater. 


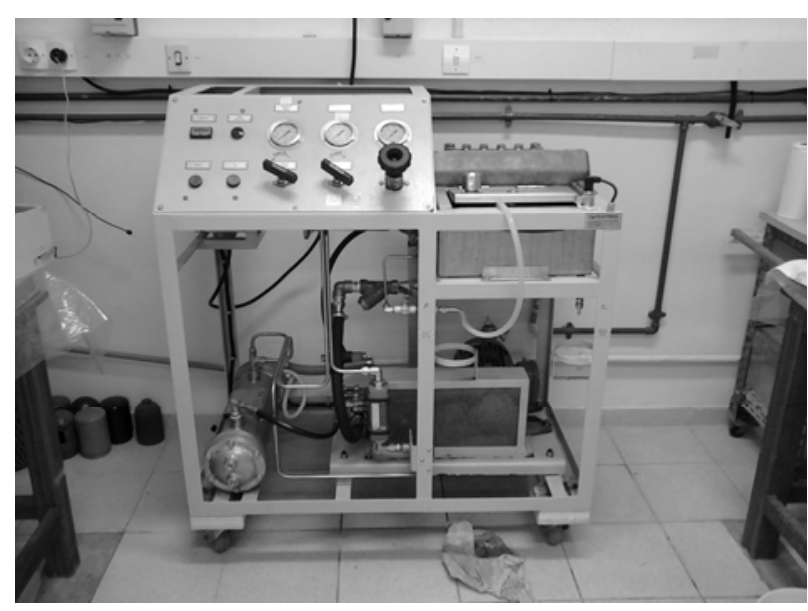

Permeation system: front view

(a)

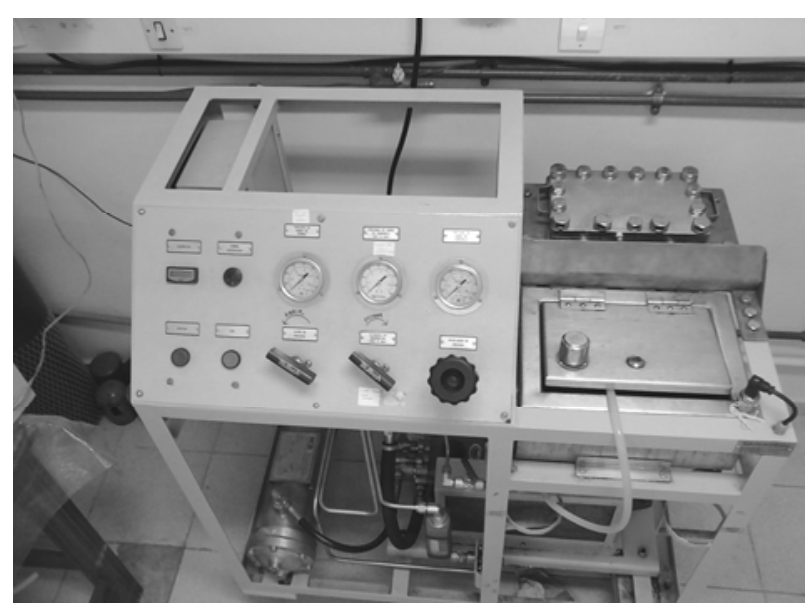

Permeation system: panoramic view

(b)

Fig. 1 Permeation system with filtration cell with displacement of the tangential flow type (brand Flutrol).

For the process using seawater plus the biocidal substance

DBNPA

(2,2-dibromo-3-nitrilopropionamide) and antifouling concentrations used in these products were $300 \mathrm{ppm}$ and 5 ppm, respectively, and the contact time was $3 \mathrm{~h}$.

\subsection{Use of Biocide and Antifouling}

In experiments with a biocide active substance containing DBNPA and an antifouling was used. The concentrations used were $300 \mathrm{ppm}$ and $5 \mathrm{ppm}$, respectively, and the contact time for both chemicals was three hours proceeding to the start of the experiments.

After the test sample is removed from the membrane cell, the system is washed with distilled water and repeats the same procedure changing the operating pressure.

\subsection{Quantifying Microbial}

\subsubsection{Planktonic Microorganisms}

The planktonic micro-organisms were quantified in water samples used in the beginning of the experiment, samples taken during the process, and samples collected after the operating time of eight days.

This procedure was performed both in the presence and absence of chemicals before starting the process.

For quantification, was inoculated $1 \mathrm{~mL}$ of the sample in sterile saline and been held decimal dilutions seriadas. The incubation was made at $37 \pm 1^{\circ} \mathrm{C}$ for $48 \mathrm{~h}$ to 15 days for anaerobic and 15 days for BRS (sulfate reducing bacteria) total aerobic bacteria. For anaerobic microorganisms media was purged with $\mathrm{N}_{2}$. We adopted the method of the MPN (most probable number) [12] for anaerobic micro-organisms and by plating technique (pour plate) for aerobic micro-organisms.

\subsubsection{Micro-sessile Organisms}

The cell count of the biofilm was performed at the end of the process, and for both conditions tested. The membrane was removed from the holder, washed with sterile removal of micro-organisms and scraped to remove non-adherent cells present in the biofilm distilled water. These cells were suspended in sterile saline and inoculated into the appropriate culture media and growth of microorganisms were performed in media specific for each group cultures conducted according to planktonic microorganisms.

\subsection{SEM (Scanning Electron Microscopy)}

The morphology of the membranes used in the experiments to check the formation of biofilm on the surface was characterized by SEM. Samples were prepared by cryogenic braking and coated a thin gold layer [13]. 


\section{Results and Discussion}

\subsection{Evaluation of the Performance of Nanofiltration Membrane}

The presence of biofouling on nanofiltration membrane causes adverse effects in the separation processes: decline of permeate flux, an increase in operating pressure (to maintain the same flow) and degradation of the membrane.

Table 1 and Table 2 show the amounts of permeated flow 15 bar for the commercial and synthesized membranes in the presence and absence of chemicals. The flow of commercial membrane showed no significant variation (Table 1). To the synthesized membrane there was a decrease in the absence of product flow may indicate the presence of scale on the surface (Table 2).

The majority of the industries that use the NF for treatment water use polyamide membranes, however, with the continued use of these membranes are formed from organic material deposition and growth of microbial communities (biofouling) on the surface causing a decrease of permeate flux and selectivity of membranes. In accordance with the data of Fig. 2 that the values of the sulfate ion rejection membrane for commercial vary slightly with the addition product, which is not observed in the synthesized membrane, where the variation is increased $[14,15]$.

One of the possibilities to lower costs with the use of scale inhibitors is somehow preventing the

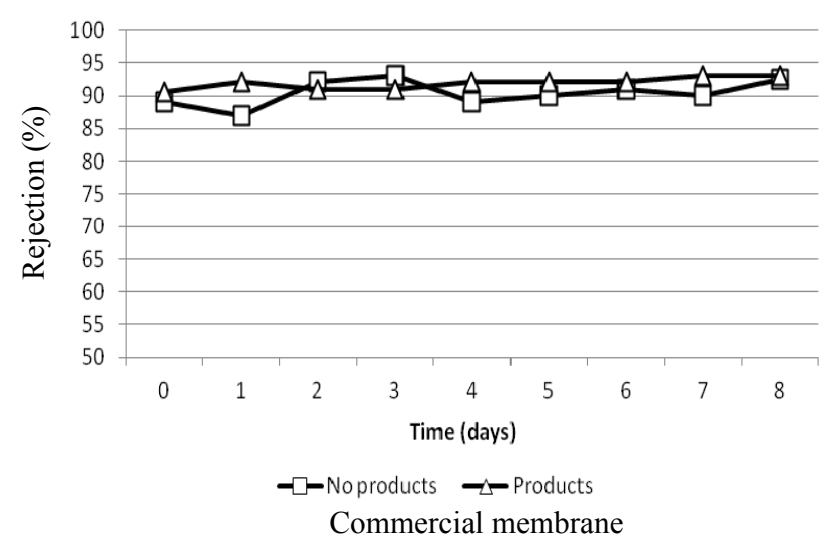

(a) possibility of deposition of salts that cause fouling, especially those with sulfates in its composition. One of the most used methods is the chemical cleaning of the membranes, they cause the process to stop the exchange of membranes cleaning occurs and when needed, causing operating costs $[16,17]$. During the filtration process with duration of 8 days, there was no exchange membrane used. The monitoring of its filtering capacity was measured by determining the

Table 1 Permeate flow $\left(L \cdot \mathrm{m}^{2} / \mathrm{h}\right)$ using a commercial membrane.

\begin{tabular}{lll}
\hline Time (days) & No products & Products \\
\hline 0 & 24.85 & 21.17 \\
1 & 22.9 & 20.15 \\
2 & 23.72 & 20.99 \\
3 & 24.23 & 21.44 \\
4 & 25.03 & 20.44 \\
5 & 22.9 & 19.85 \\
6 & 23.38 & 21.11 \\
7 & 21.29 & 19.8 \\
8 & 19.96 & 21.39 \\
\hline
\end{tabular}

Table 2 Permeate flow $\left(\mathrm{L} \cdot \mathrm{m}^{2} / \mathrm{h}\right)$ using a synthesized membrane.

\begin{tabular}{lll}
\hline Time (days) & No products & Products \\
\hline 0 & 14.82 & 14.51 \\
1 & 15.73 & 17.42 \\
2 & 13.92 & 16.31 \\
3 & 10.89 & 12.91 \\
4 & 8.17 & 12.52 \\
5 & 7.86 & 10.41 \\
6 & 8.47 & 17.93 \\
7 & 8.17 & 13.66 \\
8 & 8.77 & 14.16 \\
\hline
\end{tabular}

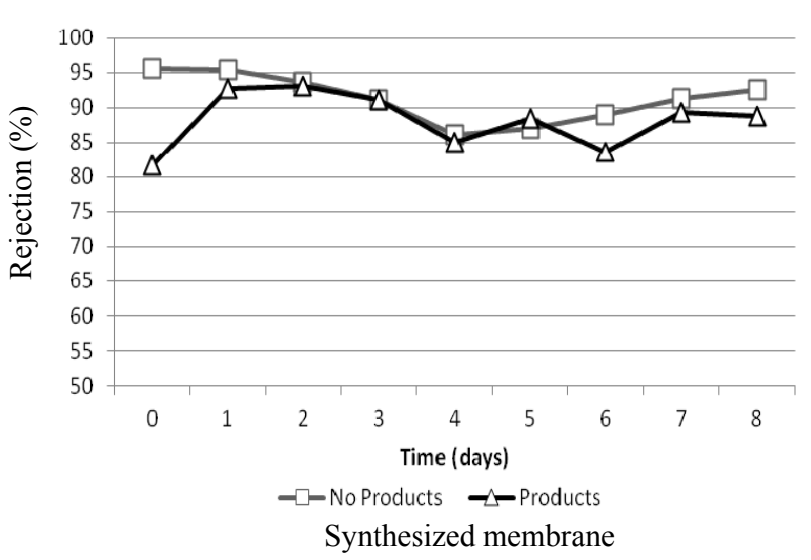

(b)

Fig. 2 Percentage of sulphates ions rejection during the performance process. 
concentration of sulfates in permeate flux. The results show that the synthesized membrane compared to the commercial again showed a shifting profile does not undergo influence of the added products (Fig. 3).

\subsection{Reviews of Microbial Growth and Biofilm} Formation on the Surface of Nanofiltration Membranes

NF membranes can have the formation of bacterial biofilms on continuous flows, and the formation and morphology of these biofilms can be related both to the properties presented on the surface as the composition of the membranes used [18].

This biomass growing on this surface can be induced by the addition of organic carbon system, and the deposition and growth of the bacterial community are able to change the charge remaining on the surface of NF membranes [19]. In the case of sea water, which contains dissolved organic material can accumulate on the membrane surface, and this layer may affect the removal of solutes with divalent ions. These fouling may increase the hydrophobicity and reduce the surface charge of some NF membranes, and the opposite effect in other can occur [20].

The surface charge of the NF membranes influences the rejection of multivalent ions and fouling resistance. Compounds with neutral charged membranes are capable of removing multivalent ions more efficiently (approximately 95\%) than monovalent ions (80\%). Moreover, the fouling could be less frequent in this type of membrane to occur as necessary for the adsorption of fouling on the surface interactions, the existing charge seems to be quite important [21].

The results with respect to the counts of planktonic microorganisms showed that regardless of the number of samples taken during the process, there was the presence of micro-organisms in permeated samples (Fig. 4). This may be due to contact with the wastewater found in the feed tank, since the permeate also returns to this tank and the collection of samples is taken from the same place of return. However, even with viable cells in permeated samples, cells quantification was lower, showing a growth inhibition of $55.6 \%$ and $68.75 \%$ for the system with the commercial membrane is synthesized, respectively, even without the addition chemicals. This result indicated that NF membrane was able to retain these microorganisms, when compared with the groups present in nature in water. Among the groups, the lower was the growth of anaerobic bacteria, which may be due to the very retention of cells by filtration or due to the environment, being under high pressure (15 bar), has a considerable flow velocity, allowing aeration of the environment.

In the analysis of quantification of existing planktonic micro-organisms in sea water early in the process and in water containing biocide and anti-fouling was observed that the addition of chemicals for a period of three contact hours before placement in the system caused reducing the amount of aerobic and anaerobic bacteria and total inhibition of growth of SRB (sulfate reducing bacteria) in both membranes tested (Fig. 5). In the process, it was observed that only the first sample there is the presence of micro-organisms, these being quantified not in the other (data not shown). This shows that the action of the biocide used is effective and that its biological action is maintained throughout the process making its application to time constants, every 2 days of operation, when water changes were carried out.

After removal of the membrane system, membranes samples were cut and these samples were used both for bacterial cells quantification of existing sessile microorganisms in the biofilm, as for the analysis in a SEM, being previously fixed in Karnowsky fixative for were the conservation of existing cells in the biofilm.

In relation to biofilm formation on the nanofiltration membrane surface, it can be observed that both in the absence and in the presence of the chemicals tested was the formation of a film on the surface of the membranes (Figs. 6 and 7). What can be 


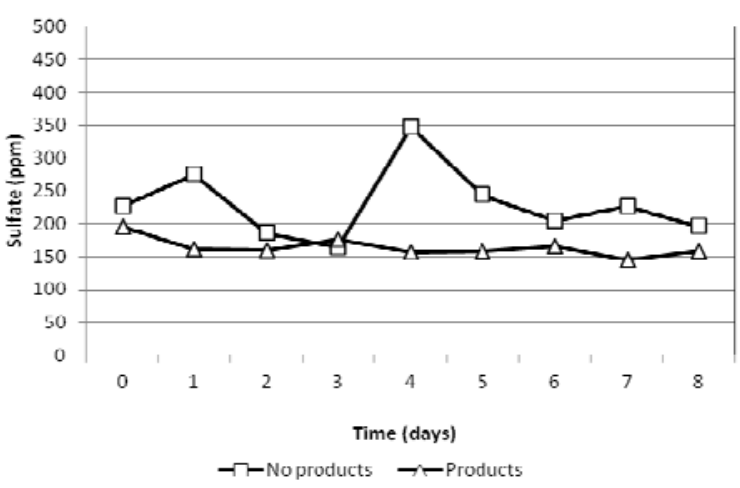

Commercial membrane

(a)

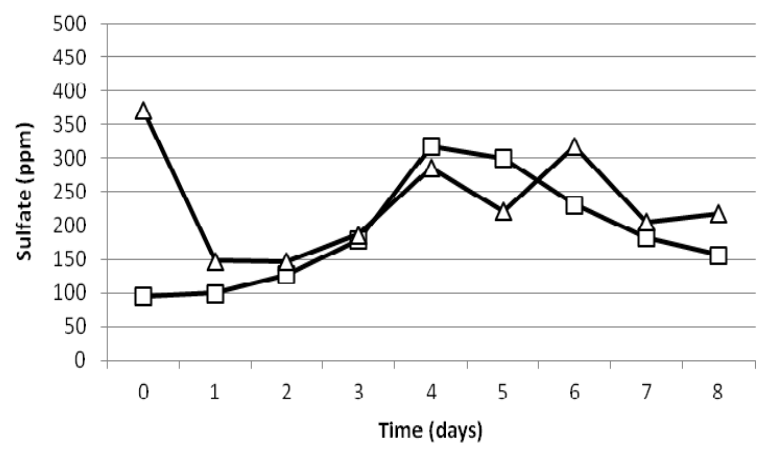

$\neg$ Noproducts $\rightarrow$ Products

Synthesized membrane

(b)

Fig. 3 Determination of the concentration of sulfate ions for performing the process.

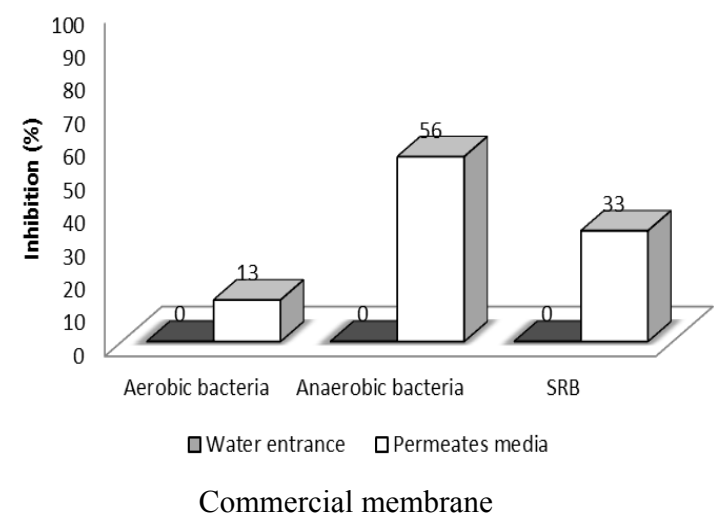

(a)

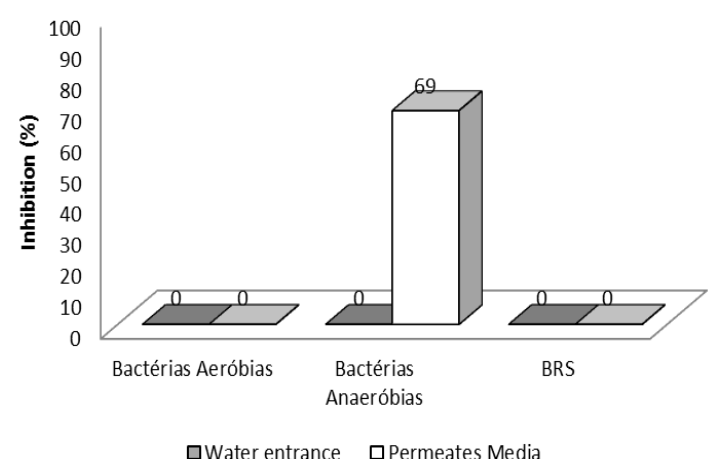

Synthesized membrane

(b)

Fig. 4 Percentage of inhibition of the growth of planktonic microorganisms in sea water during the process in the absence of biocidal and antifouling in commercial and synthesized membranes.

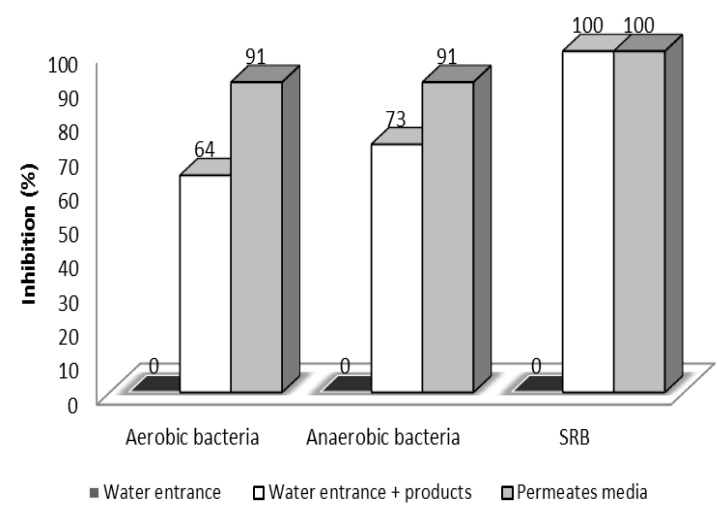

Commercial membrane

(a)

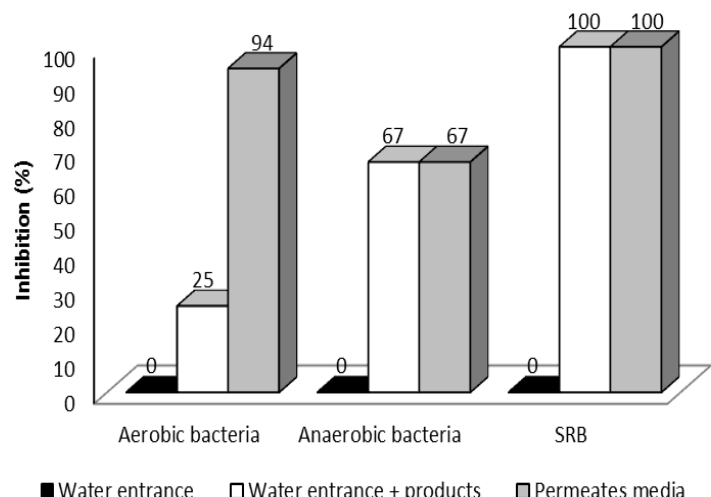

Synthesized membrane

(b)

Fig. 5 Percentage of inhibition of the growth of planktonic microorganisms in sea water during the process in the presence of biocidal and antifouling in commercial and synthesized membranes. 


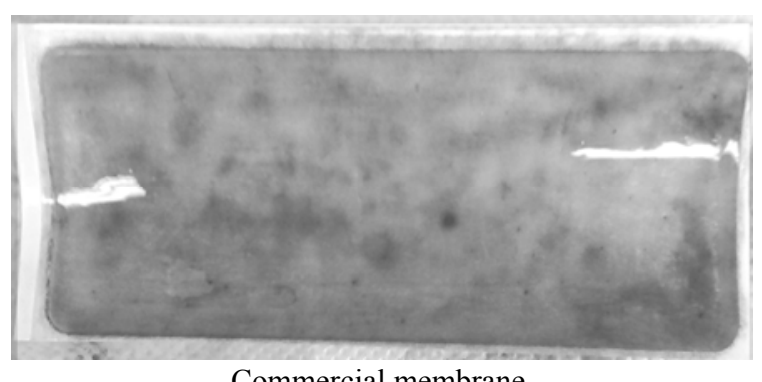

Commercial membrane

(a)

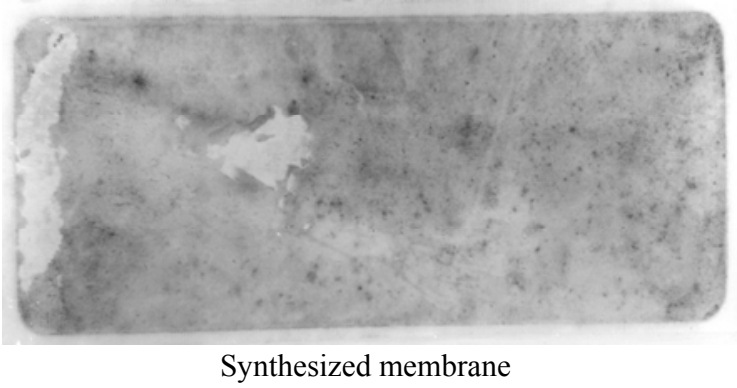

(b)

Fig. 6 Photograph of the NF membrane, after removal of the filtering system using seawater without PC-11 and biocidal antifouling PC-191T.

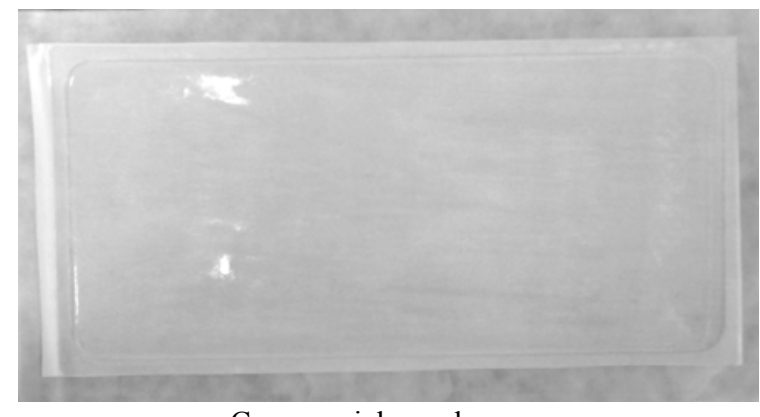

(a)

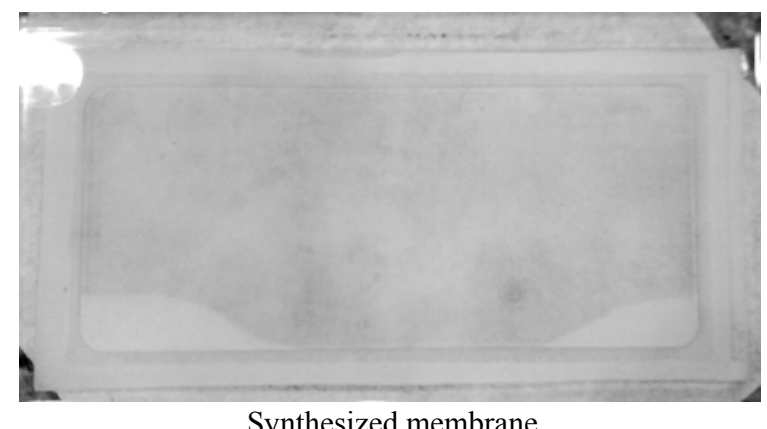

Synthesized membrane

(b)

Fig. 7 Photograph of the NF membrane after removal of the filtration system using sea water with PC-11 and biocidal antifouling PC-191T.

seen by the difference in coloration of the biofilm. In the system without the products, the formed film was presented darkness with blackened points throughout the length of both tested membranes (Fig. 6). These blackened spots are indications of BRS, which reduce sulfate to sulfide, causing a blackened precipitate. In the system the addition of the products were made, the formed film obtained was lighter shade, without the presence of blackened spots, indicating that this film did not occur in the presence of BRS (Fig. 7).

The micro-organisms present in the water treatment process that uses membranes can be transported to the surface, where adsorbed forming bio-fouling. Once adsorbed, multiply to the detriment of existing nutritional supplements in the fluid, causing reduced flow and damage the membrane. This biofilm formed by bacteria and extracellular polymers are the priority components in this structure, and the existing bacterial population converges to infinity [22].

Fig. 8, referring to the count of sessile microorganisms in the biofilms, it shows that the biocide is effective in controlling microbial growth, however, despite having acted on all bacterial groups tested appeared to be more effective in the BRS group, where inhibition occurred at $100 \%$.

This group of bacteria are the most significant because they are the main responsible for the corrosion of the material, since they are able to reduce sulfate ions to sulfide ions, causing the depolarization of the material, assisting in the corrosion process. Analyzing the composition of the biofilm formed on both types of membranes can be observed that the commercial membrane, even in the presence of biocide introduced in a biofilm consists mainly of aerobic bacteria (64\% of cells) and anaerobic (36\%). The synthesized composite membrane showed a biofilm mostly by anaerobic bacteria ( $83 \%$ of cells), and aerobic bacteria (17\%). This difference in prevalence in different bacterial groups in both membranes may be due to its composition, since for 


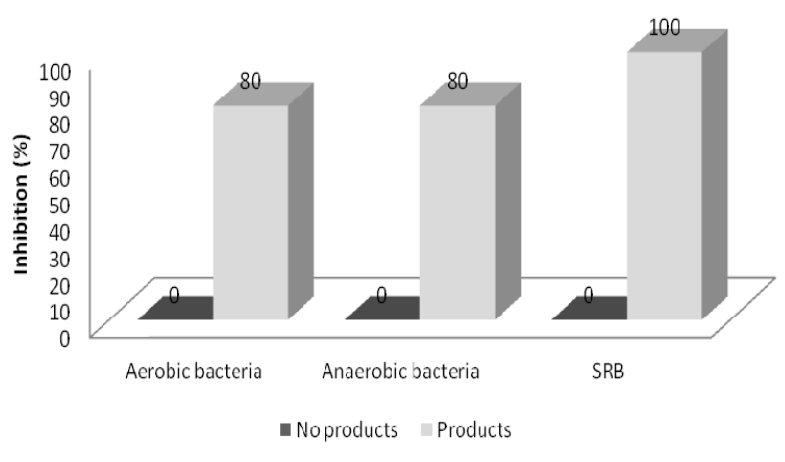

Commercial membrane

(a)

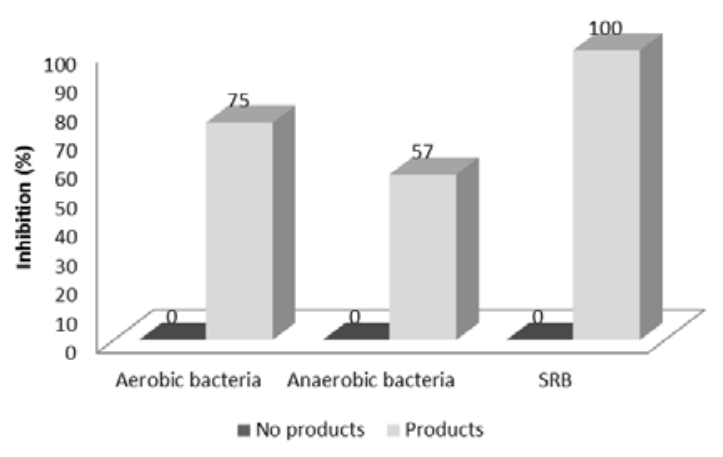

Synthesized membrane

(b)

Fig. 8 Inhibition of biofilm formation on the surface of membranes NF.

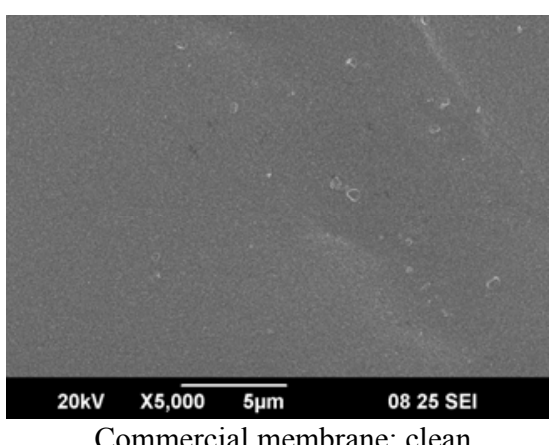

Commercial membrane: clean

(a)

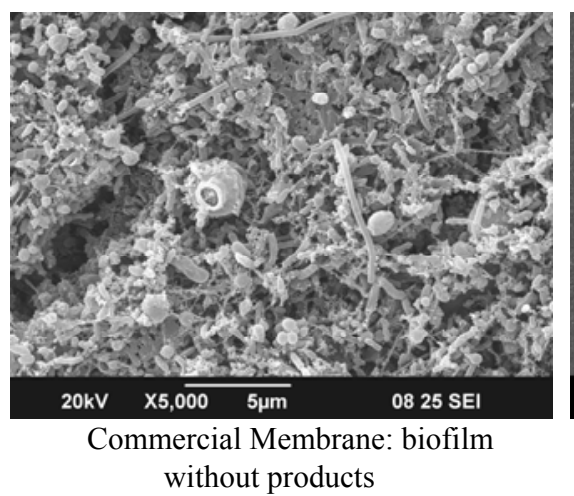

(b)

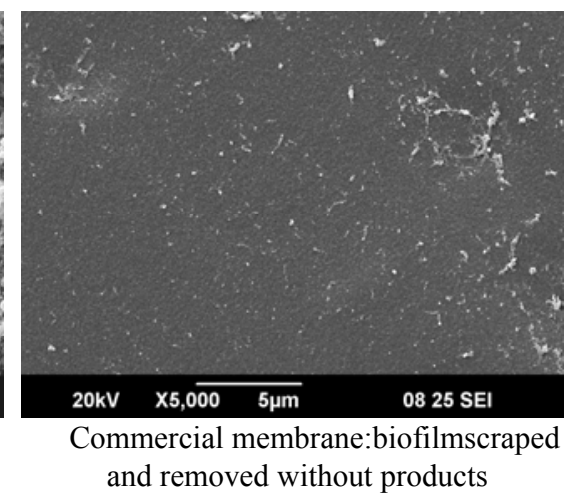

(c)

Fig. 9 SEM of the surface of the commercial NF membrane without PC-11 and biocidal antifouling PC-191T.

the formation of the biofilm, the initial step is attachment of the cells on a given support, and as shown by the presence of a polymer compound sulfonamide gave greater adhesion to the substrate of anaerobic bacteria.

Fig. 9 shows the formation of biofilms on a commercial NF membrane at the end of the 8 days of the process without the use of the biocidal substance and the antifouling. For analysis of the results, one can observe the formation of biofilm on the membrane surface. This showed dense comprising microbial cells (in the form of coconuts, long and short rods, spiral-shaped cells and the presence of micro-algae) and the formation of extracellular polymers. Also according to the results, the membrane suffered no damage to indicate degradation of the membrane due to the presence of biofilm or the use of chemical substances after the mechanical removal (scraping).
These observations were also in the analysis of the synthesized membrane (data not shown).

The biofilm formed in the presence of the biocide and anti-fouling in commercial membrane has a large amount of salt deposited and extracellular polymer produced, preventing the viewing of the microbial cells. The same effect was not observed in the synthesized membrane, where there was no such continuous training, featuring spaces containing very few cells or absence of these, there also being displayed the production of extracellular polymer (Fig. 10).

In synthesized membrane was visualized surface damage that may have been due to the use of biocide and antifouling, or even used some parameter such as pressure. This damage did not appear to affect their properties at test time ( 8 days), because the results of both permeate flux as a rejection of sulfates ions are not altered. 


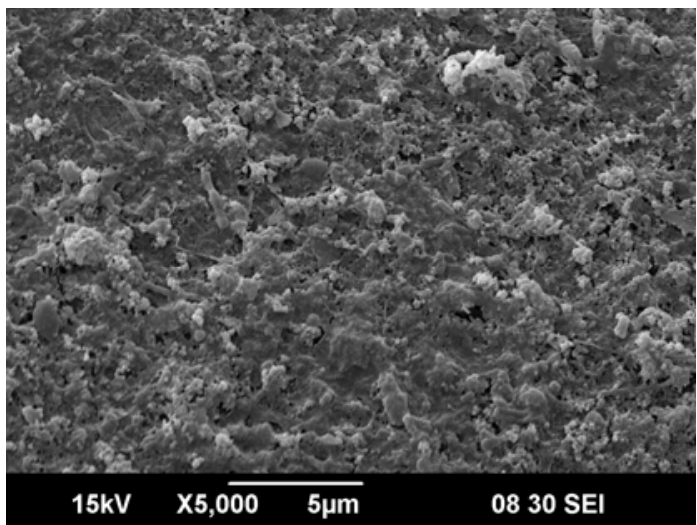

Commercial membrane: biofilm with products

(a)

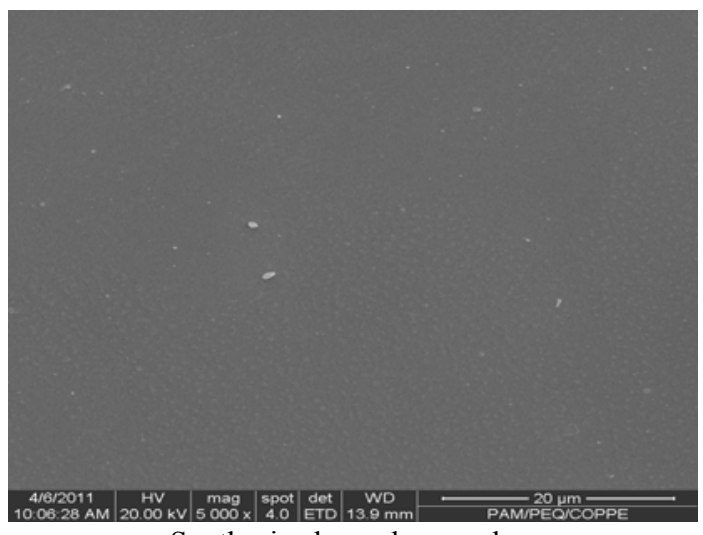

Synthesized membrane: clean

(c)

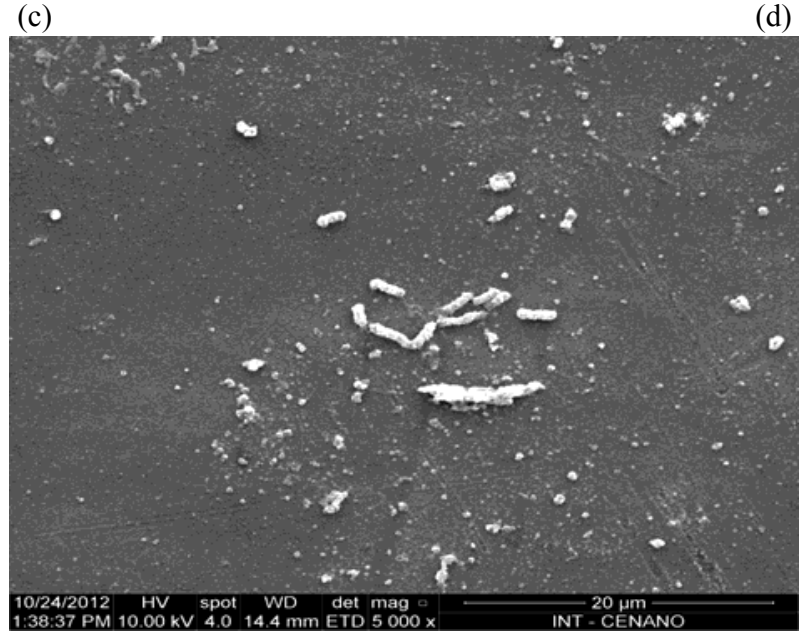

Synthesized membrane biofilm with products

(e)

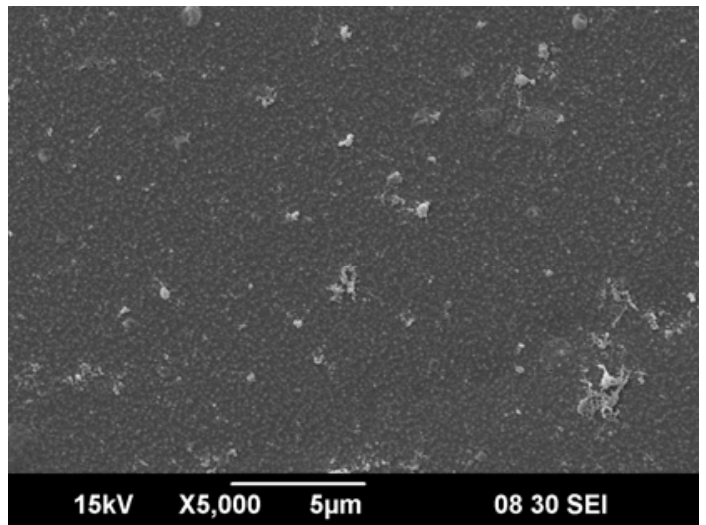

Commercial membrane: biofilm removed and products

(b)

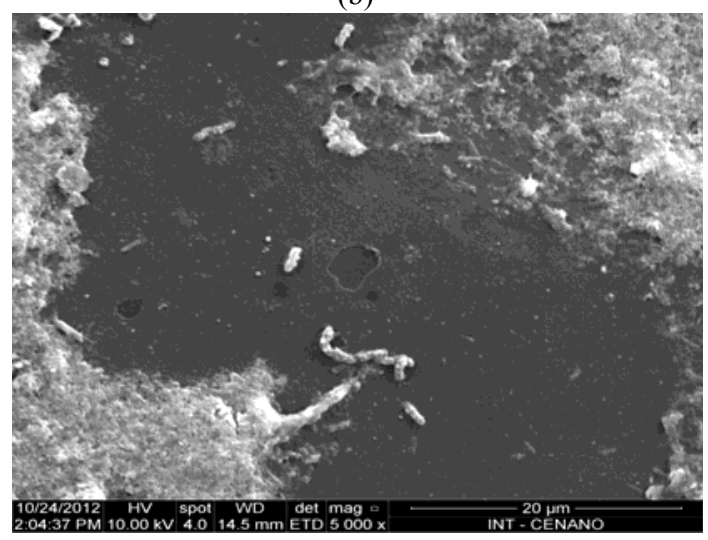

Synthesized membrane: biofilm with products

(d)

Fig. 10 SEM of the surface of NF membranes with PC-11 and biocidal antifouling PC-191T. 


\section{Conclusions}

The study of biofilm formation and use of chemical substances in NF membranes used in the oil industry to prevent bio-fouling and fouling were performed on those used in the next activity reuse of sea water for oil recovery conditions.

It was observed that the two membranes are able to withstand the chemical conditions due to the use of chemicals in the process, and physical conditions such as high pressure showing no alteration in tests proving functionality (flow values and rejection of sulfate ions). The use of biocides is feasible for the control of microbial growth on the surface of membranes, increasing their lifetime.

We conclude that NF membranes can be used for the removal of sulfates and improved recovery of petroleum without the risk of using products which cause fouling, it is chemical or biological.

\section{Acknowledgments}

The authors thank the researchers Dr Marcia Teresa Soares Lutterbach (INT-Instituto Nacional de Tecnologia) and Dr Moema de Alencar Hausen (CBPF- Centro Brasileiro de Pesquisas Físicas) for performing the technique of scanning electron microscopy.

The research described in this paper was financially supported by CNPq, CENPES/Petrobras.

\section{References}

[1] Mulder, M. 1991. Basic Principles of Membrane Technology. New York.

[2] Schafer, A. I., Fane, A. G., and Waite, T. D. 2005. Nanofiltration-Principles and Aplication. Elsevier Oxford.

[3] Mosqueda-Jimenez, D. B., Huck, P. M., and Bazu, O. D. 2008. "Fouling Characteristics of un Ultrafiltration Membrane Used in Drinking Water Treatment." Desalination 230: 79-91.

[4] Boussu, K., Van der Bruggen, B., Volodin, A., Van Haesendonck, C., Delcour, J. A., Van der Meeren, P., and Vandecasteele, C. 2006. "Characterization of Commercial Nanofiltration Membranes and Comparison with Self-made Polyethersulfone Membranes." Desalination 191: 245-253.

[5] Van der Bruggen, B., Braeken, L., and Vandecasteele, C. 2002. "Evaluation of Parameters Describing Flux Decline in Nanofiltration of Aqueous Solutions Containing Organic Compounds." Desalination 147: 281-288.

[6] Van der Bruggen, B., and Vandecasteele, C. 2001. "Flux Decline during Nanofiltration of Organic Components in Aqueous Solution." Environ. Sci. Technol. 35: 3535-3540.

[7] Peng, W. H., Escobar, I. C., and White, D. B. 2004. "Effects of Water Chemistries and Properties of Membrane on the Performance and Fouling-a Model Development Study." J. Membr. Sci. 238: 33-46.

[8] Bellona, C., and Drewes, J. E. 2005. "The Role of Membrane Surface Charge and Solute Physico-chemical Properties in the Rejection of Organic Acids by NF Membranes." J. Membr. Sci. 249: 227-234.

[9] Childress, A. E., and Elimelech, M. 2000. "Relating Nanofiltration Membrane Performance to Membrane Charge (Electrokinetic) Characteristics." Environ. Sci. Technol. 34: 3710-3716.

[10] Axel Rosenhahn, A., Sören Schilp, S., Hans Jürgen Kreuzer, H. J., and Michael Grunze, M. 2010. "The Role of Inert Surface Chemistry in Marine Biofouling Prevention.” Phys. Chem. Chem. Phys. 12: 4275-4286.

[11] Baker, J. S., and Dudley, L. Y. 1998. "Biofouling in Membrane Systems-a Review." Desalination 118: 81-90.

[12] Oblinger, J. L., and Koburger, J. A. 1975. "Understanding and Teaching the Most Probable Number Technique." J. Milk Food Technol. 38: 540-545.

[13] Mavropoulos, E., Hausen, M., Costa, A. M., Alves, G., Mello, A., Ospina, C. A., et al. 2013. "The Impact of the RGD Peptide on Osteoblast Adhesion and Spreading on Zinc-substituted Hydroxyapatite Surface." J Mater Sci: Mater Med. 24: 1271-1283.

[14] Bannoud, A. H. 2001. "Elimination of Hardness and Sulphate from Water by Nanofiltration." Desalination 137 (1-3): 133-139.

[15] Ivnitsky, H., Minz, D., Kautsky, L., Preis, A., Ostfeld, A., Semiat, R., et al. 2010. "Biofouling Formation and Modeling in Nanofiltration Membranes Applied to Wastewater Treatment.” J. Memb. Sci. 360 (1-2): 165-173.

[16] Song, Y., Su, B., Gao, X., and Gao, C. 2012. "The Performance of Polyamide Nanofiltration Membrane for Long-term Operation in an Integrated Membrane Seawater Pretreatment System.” Desalination 296: 30-36.

[17] Bader, M. S. H. 2006. "Nanofiltration for Oil-Fields Water Injection Operations: Analysis of Osmotic 
Pressure and Scale Tendency.” Desalination 201 (1-3): 114-120.

[18] Khan, M. M., Stewart, P. S., Moll, D. J., Mickols, W. E., Nelson, S. E., and Camper, A. K. 2011. "Characterization and Effect of Biofouling on Polyamide Reverse Osmosis and Nanofiltration Membrane Surfaces." Biofouling 27 (2): 173-83.

[19] Botton, S., Verliefde, A. R. D., Quach, N. T., and Cornelissen, E. R. 2012. "Influence of Biofouling on Pharmaceuticals Rejection in NF Membrane Filtration." Water Res. 46 (18): 5848-5860.

[20] Bellona, C., Marts, M., and Drewes, J. E. 2010. "The Effect of Organic Membrane Fouling on the Properties and Rejection Characteristics of Nanofiltration Membranes." Sep Purif Technol. 74 (1): 44-54.

[21] Ba, C., and Economy, J. 2010. "Preparation and Characterization of a Neutrally Charged Antifouling Nanofiltration Membrane by Coating a Layer of Sulfonated Poly (Ether Ether Ketone) on a Positively Charged Nanofiltration Membrane." J. Memb. Sci. 362 (1-2): 192-201.

[22] Ivnitsky, H., Katz, I., Minz, D., Shimoni, E., Chen, Y., Tarchitzky, J., Semiat, R., and Dosoretz, C. G. 2005. "Characterization of Membrane Biofouling in Nanofiltration Processes of Wastewater Treatment." Desalination 185 (1-3): 255-268. 